\title{
Dynamique cardiovasculaire et respiratoire
}

\author{
Compte-rendu du Colloque Euromech 92 (Compiègne 5-8 septembre 1977)
}

Cardiovascular and respiratory system dynamics

\author{
Raymond Comolet \\ Professeur à l'Université Pierre et Marie Curie, Paris VI
}

Les Colloques Euromech sont une institution européenne créée il y a une douzaine d'années ayant pour but de faire connaitre les travaux des chercheurs des différents pays d'Europe (en dehors de l'URSS) dans tous les domaines qui intéressent la mécanique.

Ils se déroulent à l'initiative d'un responsable agréé par le Comité d'Euromech, sur un sujet assez spécialisé, pouvant réunir, sur invitation, une centaine de participants parmi lesquels on compte souvent quelques représentants de pays extra européens sollicités pour leur réputation internationale. Un comité de lecture fait le choix des communications dont le nombre doit être compris entre 40 et 60 .

Ces réunions sont très appréciées par les jeunes chercheurs et leur intérêt didactique est particulièrement remarqué chaque fois qu'il est possible de demander à des personnalités scientifiques de haut niveau de présenter des exposés généraux de synthèse.

$\mathrm{La}$ variété des sujets traités dans ces colloques est assez grande pour qu'en général aucune périodicité n'apparaisse dans les titres retenus. Une exception semble cependant devoir être faite pour le colloque de biomécanique consacré à la mécanique des systèmes circulatoire et pulmonaire. Une tradition en effet s'est établie pour organiser de telles réunions tous les deux ans. Les précédentes se sont tenues à Varsovie (1971), Londres (1973) Aix-la-Chapelle (1975), et la demière en date vient de se dérouler à Compiègne, en septembre dernier. Le titre retenu à Compiègne était "Dynamique cardiovasculaire et pulmonaire", il concernait aussi bien la physiologie que le génie biomédical (mesure des paramètres mécaniques, étude d'organes artificiels et prothèses). Son intérêt se situe donc à l'interface de la physiologie et de la mécanique. Parmi les 108 participants, 46 étaient français et sur les 44 communications entendues, 16 exposaient des travaux faits en France. Depuis leur origine on a pu constater que le succès de ces "Euromech" sur la biomécanique allait en croîssant et les chiffres précédents montrent bien que cette discipline, largement développée dans les pays anglosaxons, s'est bien implantée dans le nôtre. Enfin, occurence exceptionnelle, les communications de ce dernier Euromech seront publiées in-extenso par les soins de I'INSERM.

Dans ce qui suit, je me propose de donner un bref aperçu des travaux de ce colloque.

Les conférences et communications se groupent autour des thèmes suivants:

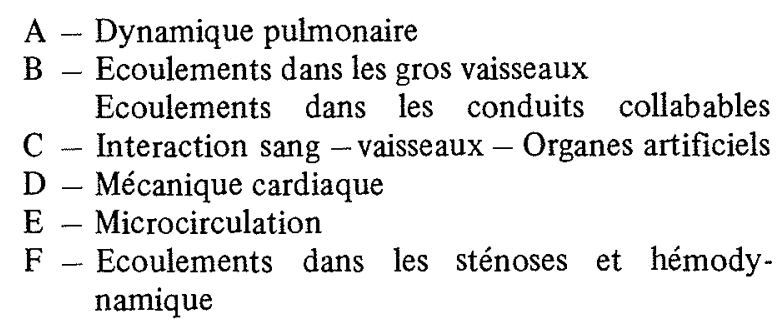

\section{Dynamique pulmonaire}

La première séance de travail commence par une conférence de P.T. Macklem [1] sur certains aspects de la physiologie des voies respiratoires.

On sait depuis longtemps que la chute de pression, le long de l'arbre pulmonaire, se situe principalement dans les voies supérieures (proches du larynx) alors que les voies inférieures (périphériques) qui concement des conduits de diamètre inférieur $3 \mathrm{~mm}$ environ, participent surtout aux échanges gazeux entre l'air inspiré et le gaz alvéolaire. Mais on admet que ces conduits présentent une certaine instabilité de forme qui peut conduire à leur fermeture aux faibles valeurs du volume pulmonaire.

En cas de maladie, la zone principalement responsable de la chute de pression peut se déplacer des voies supé- 
rieures vers les voies inférieures de sorte que la fermeture des conduits peut apparaitre pour des volumes pulmonaires plus importants. Le mécanisme de cette fermeture est encore inconnu, comme l'est aussi le rôle joué dans la régulation de la fonction respiratoire par les petits muscles bronchiques.

Viennent ensuite 3 communications anglaises.

La première, de S. Cameron [2], est une étude de la forme des alvéoles pulmonaires au cours de la respiration. Par injection de résine polyester dans des poumons d'agneaux, on peut étudier le volume pulmonaire à différents stades de gonflement et, après dissolution chimique des tissus biologiques, examiner au microscope la forme prise par les alvéoles. Cette forme évolue entre le discoïde et la sphère en passant par l'ellipsoïde. A partir de ces formes il est possible de déterminer une courbe tension-déformation qui s'est révélée analogue à celle des autres tissus vivants, tel que la peau.

Un problème qui préoccupe depuis longtemps les physiologistes de la respiration, est celui du mélange des gaz dans les conduits pulmonaires: dans la communication de K. Horsfield et al. [3], on aborde ce problème en étudiant la vitesse d'avancement du front d'un mélange gazeux de $\mathrm{SF}_{6}-\mathrm{H}_{\mathrm{e}}$ ou $\mathrm{SF}_{6}-\mathrm{A}_{\mathrm{r}}$ dans un arbre bronchique en résine, préalablement rempli d'air, et dans celle de A.E. Bunn, et al. [4] on analyse la composition en $\mathrm{CO}$, au cours du cycle respiratoire, d'un mélange respiré contenant $0,04 \%$ de $\mathrm{CO}-$ Dans cette analyse des gaz, beaucoup de difficultés techniques doivent être surmontées pour obtenir un "indice du degré de mélange" - La communication d'un jeune chercheur français, G. Ellenberger [5] traite ensuite des pertes de charge, dans un réseau à bifurcations multiples: il s'agit d'un réseau plan usiné dans une plaque de plexiglas comportant 6 bifurcations successives, donc $2^{6}=64$ sorties dans une distribution géométriquement symétrique et présentant quelques analogies dimensionnelles avec un compartiment du réseau pulmonaire. Contrairement à toutes les études antérieures sur les écoulements à travers des bifurcations (où les modèles ne présenteraient que 1 ou 2 bifurcations successives), une telle disposition permet de mettre en évidence l'interaction d'une singularité (la bifurcation) sur la suivante. Dans ce modèle on peut étudier l'écoulement dans une conformation aussi bien divergente que convergente. Dans le premier cas par exemple (inspiration) on met en évidence une certaine hierarchie dans les voies suivies par le fluide, pour traverser le réseau. Ainsi l'orifice de sortie qui laisse passer le débit le plus élevé correspond au cheminement comportant le maximum de changements de direction alternés. Cette hierarchie semble disparaître dans le mode convergent (expiration)

\section{Ecoulements dans les gros vaisseaux}

$\mathrm{Ce}$ thème a sûrement fait l'objet du plus grand nombre d'études de la part des mécaniciens et la conférence du Professeur E. Wetterer et al [6] sur la dynamique artérielle brosse un large tableau d'ensemble sur les théories de la dynamique circulatoire artérielle. Les phénomènes de propagation et réflexion d'ondes, sont décrits en termes de célérité et d'impédances. Les modèles artériels sont nombreux, linéaires ou non, à caractéristiques réparties ou non, électriques, mathématiques. Le caractère viscoélastique de la paroi déformable des vaisseaux, ne peut plus être négligé et ajoute une complication considérable tant pour les mesures expérimentales que pour les développements théoriques. Un de ces modeles à paramètres discrètement répartis est étudié par M. Anliker et al [7], par la méthode des caractéristiques et celle des différences finies. On y tient compte de la présence éventuelle de shunts ou de sténoses.

Un groupe de recherche de biomécanique très important se trouve à Aix-la-Chapelle, dirigé par le Professeur Krause, il va fournir plusieurs communications. La première de Nan-suey Liu [8], fait l'étude théorique complète d'un écoulement visqueux dans une conduite circulaire courbe, modèle simplifié de la crosse aortique. L'intérêt de ce travail est de calculer la contrainte visqueuse à la paroi, dont le rôle est très important en pathologie. Toutefois le nombre de Reynolds de 200 retenu par l'auteur pour développer ses calculs n'est guère représentatif de celui qui existe au niveau de l'aorte chez l'homme. Cette aorte fait en effet l'objet de beaucoup d'attention de la part des chercheurs. Dans sa communication A.J. Sobey [9] s'intéresse à la stabilité du mouvement du sang dans ce vaisseau, stabilité étudiée dans un modèle de tuyau rectiligne dont l'écoulement est sollicité par un gradient de pression de la forme $\frac{\partial p}{\partial x}=A+B \cos \omega t$. Dans la communication de D.L. Newman et al. [10], on mesure directement chez le chien, et dans différentes situations physiologiques, la célérité des ondes pulsatiles dans l'aorte, et on en compare la valeur avec celle qu'on peut calculer en élasticité classique. Cette comparaison est très intéressante car la célérité des ondes reflète en quelque sorte les propriétés mécaniques de la paroi vasculaire. La déformation du vaisseau au passage de l'onde est relevée au moyen d'une jauge à mercure (tube de silastic rempli de mercure et ceinturant le vaisseau).

\section{Ecoulements dans les tuyaux collabables}

Est collabable une conduite susceptible de s'écraser plus ou moins (et d'une manière reversible) sous l'action de la pression extérieure. Le mouvement des fluides dans de telles conduites a fait l'objet de très peu d'études fondamentales et cependant il bénéficie de propriétés intéressantes, par exemple la célérité de propagation des ondes de pression peut s'abaisser à des valeurs 100 à 1000 fois inférieures à celles qu'on observerait si le tuyau était rigide. Les veines, certaines artères, l'urêtre sont des conduits collabables. Deux communications traitent de cette question : celle de R. Comolet [11] qui expose quelques résultats généraux concernant la nature de l'écoulement, la stabilité et la forme en long du tuyau ; celle de M. Bonis et C. Ribreau [12] qui établissent la loi de déformation de la section du conduit sous l'action de la pression par voies théorique et expérimentale. 


\section{Interaction sang-vaisseaux et organes artificiels}

Une conférence générale sur l'écoulement à travers les parois capillaires est donnée par C.C. Michel [13]. Les mécanismes de transport transmembranaire sont encore mal connus; ils sont différents selon qu'il s'agit d'eau et de molécules hydrophiles ou de macromolécules.

En pathologie et en physiologie on s'intéresse beaucoup aux interactions sang et parois artérielles afin de saisir les processus qui entrainent la formation par exemple de dépôts athéromateux obturant plus ou moins la lumière du vaisseau. Ce problème a été abordé dans plusieurs communications. Dans celle de R.M. Nerem et al. [14], on étudie l'influence des lésions provoquées sur animal par des vibrations extérieures imposées, des mesures sont faites in vivo au moyen d'anémomètres à film chaud à partir desquelles on espère obtenir des indications sur les contraintes visqueuses pariétales responsables sans doute des altérations observées. Dans celle de A. Baldwin et al. [15], on étudie les effets de la pression transmurale sur le développement initial des athéromes. La méthode consiste a examiner au microscope la progression à travers la paroi artérielle d'un traceur chimique caractérisant les cellules endommagées. Ces expériences sont faites sur animal et comparées avec d'autres essais utilisant des segments vasculaires isolés. Ainsi la communication de S. Weinbaum [16] décrit le mécanisme de transport par vésicules des macromolécules à travers l'endothelium artériel, mécanisme faisant intervenir le mouvement brownien.

Plusieurs communications étudient le fonctionnement de certains organes artificiels et les améliorations possibles. Ainsi pour B.J. Bellhouse et al. [17] on pourrait améliorer les performances des hémodialyseurs en utilisant des surfaces d'échanges corruguées et un écoulement pulsé pour le sang à dialyser. Les mouvements tourbillonnaires dans un sens et dans l'autre prenant naissance dans les sillons, peuvent multiplier les transferts par un facteur égal à trois. Un film illustre très clairement l'intérêt de cette méthode. Quant à T.V. How and J.D.S. Gaylor [18], ils etudient la dynamique du sang dans les conduits capillaires des hémodialyseurs, portant leur attention sur le temps de séjour dans l'appareil des hématies et celui du plasma au moyen d'une technique de radiotraceurs. Comme cela était d'ailleurs déjà connu, on observe dans l'écoulement une redistribution des hématies fonction de l'hématocrite. G. Heuser et B. Trossky [19] recherchent la meilleure forme à donner à une pompe d'assistance cardiaque pour que l'écoulement ne présente pas de zone morte et où soit réduit le plus possible le contact entre fluide et paroi. Trente formes de ventricules ont été étudiées sur une table à eau. Le mouvement de l'eau est visualisé par des particules en suspension ou par colorimetrie au moyen d'un liquide coloré injecté. Le caractère tridimensionnel des mouvements apparaît dans un film. J. Köhler [20] étudie la dynamique d'une valve cardiaque artificielle, en l'occurence une valve de Starr, petite sphère dans laquelle on a noyé un microaccéléromètre. Les essais ont été effectués avec un modèle élastique du cœur gauche. Pendant la plus grande partie de l'ejection systolique, la sphère resterait, d'après l'auteur, dans une position intermédiaire à l'intérieur de la cage qui en limite les déplacements.

\section{Mécanique cardiaque}

La mécanique cardiaque peut être étudiée d'un point de vue théorique ou la dynamique du sang s'exprime en termes d'impédances: c'est ce que développent T.J.Pedley et W.A. Seed [21] dans leur communication, mais ils examinent le problème en se plaçant du côté cœur, et non plus du côté circulation systémique comme on a 1habitude. Toutefois l'interaction entre ventricule gauche et système artériel est étudiée par T.J. van der Werff et Lyn A. Hanmer [22], au moyen d'un modele mathématique numérique standard sur lequel on peut introduire des perturbations diverses: variations de la résistance périphérique, du volume systolique, de la vitesse de contraction cardiaque etc... Les résultats sont conformes à ceux qu'on observe en physiologie clinique. C. Oddou et al. [23] présentent une très belle analyse du mouvement de la valve mitrale chez l'homme, fondée sur une recherche clinique en cinéangiographie et echocardiographie complètée par une étude théorique sur un modèle simplifié axisymétrique reproduisant bien la forme de la valve au cours de ses déplacements et mettant en évidence dans le fluide la distribution tourbillonnaire observée expérimentalement dans le ventricule. N. Talukder et al. [24] étudient la mécanique de fermeture de la valve aortique sur un modèle physique, la visualisation étant faite par bulles d'hydrogène ou par billes de plastique. J.P. Merillon et al [25] recherchent les facteurs déterminants le volume télésystolique ventriculaire gauche; il s'agit d'un travail fait en clinique sur des patients traités avec diverses drogues. P.J. Hunter [26] expose une méthode expérimentale de mesure des propriétés du muscle cardiaque en contrôlant optiquement le mouvement de deux fines aiguilles de $20 \mu$ de diamètre, implantées à $0,5 \mathrm{~mm}$ l'une de l'autre, dans une préparation musculaire isolée soumise à stimulation. Une tentative d'évaluation de la contractilité globale du ventricule gauche est développée par G. Demoment et J. Hinglais [27] au moyen d'un modèle mathématique fondé sur la notion d'élastance systolique maximale. D. Kalmanson et al [28] décrivent une méthode d'analyse non sanglante utilisée en clinique pour faire des explorations profondes : valves cardiaques, artères pulmonaires, aorte etc... et relever en particulier les profils de vitesses du sang. Dans cette méthode on associe l'échographie avec le vélocimètre Doppler pulsé. Toujours en application clinique, P. Besse et al. [29] décrivent le système Syscomoram qui permet, à partir de différents signaux biologiques, d'analyser en temps réel les paramètres hémodynamiques et d'évaluer les performances ventriculaires.

\section{Microcirculation}

S.P. Sutera [30] est un spécialiste de la circulation capillaire. On lui doit une très intéressante conférence 
sur la mécanique des hématies dans les petits vaisseaux. Dans les plus petits, cette mécanique est compliquée par la déformation importante que subissent les hématies, ce qui conduit à une relation pression-débit non linéaire. Cette déformation cellulaire est observée au microscope par Th. Fischer et al. [31] sur des hématies placées dans une solution de dextran, placée dans un viscosimètre cone-plaque, réalisant ainsi un intense champ de contraintes visqueuses. Dans ces conditions la membrane cellulaire subit un mouvement périodique que rend visible de petites particules servant de marqueurs. Un film montre le ballet extraordinaire exécuté par ces particules dont la fréquence dépend du taux de cisail. lement du milieu. Dans un champ de contrainte de cette nature, la membrane des hématies semble tourner autour de son contenu liquide constitué d'hémoglobine. Pour obtenir une meilleure compréhension de ce mécanisme étrange, D. Barthes-Biesel et al. [32] étudient par voie théorique le comportement d'une cellule sphérique dans un tel champ et les déformations qui s'ensuivent et retrouvent qualitativement celui qu'on observe expérimentalement. Mais les hématies, normalement de forme discoïdale, peuvent se grouper face à face en formant des rouleaux. La formation d'un rouleau est discutée par $\mathrm{R}$. Skalak et al. [33] comme un problème d'adhésion de fines membranes élastiques et sa destruction comme un problème de fracture mécanique. Les calculs sont en accord avec les observations expérimentales.

L'écoulement du sang dans les vaisseaux de diamètre inférieur à $100 \mu$ manifeste des propriétés imprévues puisqu'on constate que la viscosité apparente décroît en même temps que le diamètre du conduit (effet Fahraeus-Lindquist). Ce phénomène est connu depuis longtemps, et est lié à la présence d'une couche plasmatique pariétale pauvre en hématies. Un modèle d'écoulement satisfaisant est proposé par V. Seshadri et M.Y. Jaffrin [34] où les deux zones présentent des comportements différents. T. Kenner et al. [35] présentent un densimètre permettant de faire des mesures en continu in vivo. L'appareil est un résonateur mécanique dont on mesure la fréquence de résonance. L'appareil a été utilisé pour étudier les variations de densité du sang consécutives à l'absorption de drogues diverses, notamment au niveau du lit capillaire au cours de petites hémoragies. A. Syrota et al. [36], déterminent les coefficients thermodynamiques qui interviennent dans les échanges à travers les membranes capillaires du poumon, et leurs variations avec le poids moléculaire du soluté.

\section{Ecoulements dans les sténoses et hémodyna- mique}

Une conférence est faite par P. Peronneau [37], où l'auteur décrit l'appareil qu'il a mis au point et qui est bien connu maintenant des hémodynamiciens. C'est un vélocimètre Doppler à ultra-sons, fonctionnant sur un mode pulsé au moyen duquel on peut établir des profils de vitesses instantanées le long d'un diamètre vasculaire et déterminer l'état d'agitation du fluide (turbulence). Un film d'animation illustre bien les possibilités de la méthode, en donnant les profils de vitesses en différentes sections de l'aorte et leur évolution au cours du cycle cardiaque. La méthode est appliquée par M. Siouffi et al. [38] pour étudier l'influence d'une sténose sur la structure de l'écoulement sanguin, les essais étant faits in vivo et in vitro sur un modèle physique. C. Clark [39] s'intéresse à la turbulence produite par une sténose aortique ; il fait des mesures à l'anémomètre à film chaud sur un modèle physique tubulaire et détermine le spectre de puissance du signal. La communication de D. Tomm [40] concerne le bruit produit par la sténose toujours sur des modèles de géométries différentes. L'analyse permet de distinguer les fréquences qui sont dues aux fluctuations de l'écoulement de celles qui proviennent des vibrations propres de la paroi. Le problème de la sténose a fait aussi l'objet de plusieurs études théoriques, ainsi celle de J. Doffin et al. [41] qui est menée dans la perspective d'un écoulement sinusoidal. Le nombre de Reynolds est compris entre 5 et 60 et le paramètre de fréquence $\alpha=\mathrm{R} \sqrt{\frac{\omega}{\nu}}$ entre 1 et 10 (dans l'aorte humaine $\alpha$ est de l'ordre de 15). L'auteur met en évidence un ou deux tourbillons toriques qu'une visualisation ultérieure à permis de reconnaître. Un autre calcul est présenté par B. Forestier et al. [42] pour analyser l'écoulement oscillant laminaire dans un canal bidimensionnel plan en présence d'un obstacle pariétal de section carrée. La paroi artérielle elle-même se comporte comme un corps viscoélastique dont les modules d'élasticité ne sont pas constants. H. Schwerdt et A. Constantinesco [43] présentent un modèle qui permet de calculer la célérité de l'onde pulsatile, la déformation de la paroi, l'impédance hydraulique et quelques autres paramètres. La distensibilité radiale semble le facteur le plus intéressant d'un point de vue clinique; il est possible de la déterminer directement au moyen d'un catheter spécial. Les conséquences hémodynamiques d'une intervention chirurgicale peuvent être examinées de différentes façons: par W. Sandmann et al. [44] au moyen du vélocimètre Doppler pulsé, en analysant le profil des vitesses et la turbulence de l'écoulement sanguin, notamment après une anastomose ; par P.A. Butler et P.G. Sommerville [45] en exploitant la notion d'impédance, ce qui permet de caractériser globalement le comportement du lit vasculaire selon les changements géométriques et mécaniques dont il est le siège ; par B. Masson et al. [46] qui décrivent les modifications du retour veineux d'un membre sous l'influence d'un shunt artério-veineux, traitement appliqué à l'homme en cas de thrombose veineuse.

Ce tour d'horizon, peut-être un peu monotone, montre bien la variété des sujets abordés dans cette manifestation scientifique, et beaucoup pourraient sûrement prendre place dans les sciences de l'ingénieur.. nous en verrons quelques uns au cours des deux journées qui viennent.

Enfin, pour illustrer l'intérêt que continue de susciter ce colloque de dynamique cardio vasculaire et pulmonaire dans le cadre des Euromech, on peut annoncer que déjà trois pays se sont proposés pour organiser les prochains. 
Bibliographie

[1] MACKLEM P.T. - Physiology of the Peripheral Airways. Laboratoire d'Explorations Fonctionnelles. Hôpital Laënnec, Paris, et Meakins-Christie Laboratories, Royal Victoria Hospital, Montréal.

[2] CAMERON S. - Geometric changes of lung alveoli during inflation : implications on a stress analysis of alveolar walls. - Sussex Center for Medical Research - Brighton, England.

[3] HORSFIELD K., DAVIES A., CUMMING G. - Separation of Gas Mixtures in the Conducting Airways. Midhurst Medical Reasearch Institute - Midhurst West, Sussex, England.

[4] BUNN A.E., LEVER M.J., SCHROTER R.C. - The Mixing of Inspired and Residual Gas in the Lung. - Physiological Flow Studies Unit, Imperial College, England.

[5] Ellenberger G. - Pertes de Charges dans un Réseau à Bifurcations Multiples : Application à l'Ecoulement Pulmonaire. - Université Pierre et Marie Curie - Laboratoire de Mécanique Expérimentale des Fluides - Bât. 502 - Campus d'Orsay - 91405 Orsay

[6] WETTERER E., BAUER R.D., BUSSE R. - Arterial Dynamics. Institut für Physiologie und Kardiologie der Universität, Waldstrasse 6, D-8520 Erlangen, Germany.

[7] ANLIKER M., STETTLER J.C. et NIEDERER - Theoretically Predicted Flow and Pressure Pulses in Human Arterial Conduits. Institute for Biomedical Engineering, University of Zurich, The Swiss Federal Institute of Technology, Zurich, Switzerland.

[8] NAN-SUEY LIU - Developing Flow in a Curved Pipe. - Aerodynamisches Institut der RWHT Aachen, Aachen, Germany

[9] SOBEY A.J. - Stability of Aortic Blood Flow. - Physiological Flow Studies Unit, Imperial College, London SW7

[10] NEWMAN D.L., GREENWALD S.E. et BOWDEN N.L.R. - Comparison Between Theoritical and Directly Measured Pulse Propagation Velocities in the Aorta of the Anaesthetised Dog. - Physics Dept. - Guy's Hospital Medical School - London Bridge SE 1-England.

[11] COMOLET R. - Sur la Nature et la Stabilité de l'Ecoulement Unidimensionnel dans une Conduite Déformable Elastiquement. Laboratoire de Mécanique Expérimentale des Fluides. Université Pierre et Marie Curie - Paris.

[12] BONIS M., RIBREAU C. - Pressure-Flow Relationships in Collapsible Tubes. - Dept. de Génie Biologique Université de Technologie de Compiègne - Laboratoire de Mécanique Expérimentale des Fluides, Bât. 502, 91405 Orsay

[13] MICHEL C.C. - Flow Through Capillary Walls. - University, Laboratory of Physiology, Oxford OX1 3PT, U.K.

[14] NEREM R.M., CORNHILL J.F., KARAYANNACOS P.E., PANTALOS G.M. et RITTGERS S.E. - Hemodynamics and the Arterial Wall. - Cardio-Pumonary Research Group. The Ohio State University, Columbus, Ohio 43210

[15] BALDWIN A., WINLOVE C.P., DAVIS J. et CARO C.G. - The Effect of Pressure on The Uptake of Tracer Materials by the Rabbit Thoracic Aorta. - Physiological Flow Studies Unit, Imperial College, London, S.W. 7.

[16] WEINBAUM S. - On the Attachment, Detachment and Brownian Motion of Plasmalemma Vesicles in Arterial Endothelium. - Dept. of Mechanical Eng'g. The City College of the City University of New-York, 140 St. and Convent Ave. New-York. N.Y. 10031.

[17] BELLHOUSE B.J., PHIL D. - Augmented Mass Transfer in a Membrane Lung and a Haemodialyser Using VortexMixing. - Department of Engineering Science, Oxford University, Parks Road, Oxford, U.K.

[18] HOW T.V. et GAYLOR J.D.S. - Haemodynamic Characteristics of Parallel Flow Haemodialysers. - University of
Strathclyde, Bioengineering Unit, Wolfson Centre, 106 Rottenrow, Glasgow G4 ONW.

[19] HEUSER G. et TROSSKY B. - Flow Conditions in blood Leading Parts of Blood Pumps. - Aerodynamisches Institut der RWHT Aachen, Aachen, Germany.

[20] KOHLER J. - Fluid-Dynamical Forces on Spherical Occluders in Prosthetic Aortic Valves. - Aerodynamisches Institut der RWHT Aachen, Aachen, Germany.

[21] PEDLEY T.J. et SEED W.A. - Fluid Dynamics of Left Ventricular Ejection. T.J. PEDLEY, Department of Applied Mathematics and Theoretical Physics, Cambridge University et W.A. SEED, Department of Medicine, Charing Cross Hospital, London.

[22] Van der WERFF T.J. et HANMER L.A. - The Dynamic Interaction of the Left Ventricle and Systemic Arteries. - Deparment of Bioengineering, University of Cape Town and Groote Schuur Hospital, Cape Town, South Africa.

[23] ODDOU C. BRUN P. BERALDO F. KULAS A. et de VERNEJOUL F. - Fluid Mechnics in the Human Left Ventricle During Cardiac Filling Phase. - Laboratoire de Biorhéologie et d'Hydrodynamique - Université Paris VII et Serv. de Physiologie et d'Exploration Fonctionnelle (Groupe de Recherche U 138 INSERM) CHU Henri Mondor - 94000 Creteil.

[24] TALUKDER N., REUL H. et MULLER E.W. - Fluid Mechanics of the Natural Aortic Valve. - HelmholtzInstitut for Biomedical Engineering at the RWHT Aachen, 51 Aachen, Goethestr. 27/29, W Germany

[25] MERILLON J.P., MOTTE G. et GOURGON R.-Contribution à l'Etude des Determinants du Volume Telesystolique Ventriculaire Gauche. - Service de Cardiologie - Hôpital Beaujon 100 Bd Gl Leclerc - 92110 Clichy

[26] HUNTER P.J. - Experimental Measurement of Cardiac Muscle Properties. - Oxford University, Dept. Eng'g Science, Oxford.

[27] DEMOMENT G. et HINGLAIS H. - Evaluation of Cardiac Contractility Trough Identification of a Mathematical Model Founded on Maximal Systolic Elastance. - G. DEMOMENT, Laboratoire des Signaux et Systèmes (CNRS/ESE) Ecole Supérieure d'Electricité - Plateau du Moulon - 91190 Gif-sur-Yvette, et J. HINGLAIS, Centre d'Etudes des Techniques Chirurgicales (CNRS) Hôpital Broussais - rue Didot - 75014 Paris.

[28] KALMANSON D., VEYRAT C., ABITROL G. et BOUCHAREINE F. - Non Invasive Recording of Blood Velocity Patterns in the Heart Cavities and Large Vessels - The Department of Cardiology, Fondation A. de Rotschild, Paris

[29] BESSE P., GOUVERNEUR G., LE GOFF et CASQUeVAUX J. - Analyse des Paramètres Hémody. namiques en temps réel dans le Système SYSCOMORAM. - Dr. P. BESSE, Centre de Cardiologie - Hôpital du Tondu, 33076 Bordeaux Cedex.

[30] SUTERA S.P. - Red Celle Motion and Deformation in the Microcirculation. - Department of Mechanical Engineering, Washington University, St Louis, M063130

[31] FISCHER Th., SCHMID-SCHONBEIN H. et STOHR M. Mechanical Behaviour of Human Red Blood Cells (RBC) in the Shear Field of Viscous Dextran Solutions (DXS) (with film). - Abt. Physiologie - Melatener Str: 211 D 5100 Aachen.

[32] BARTHES-BIESEL D., GUERLET B. et STOLTZ J.F. Deformation of a Sphered Red Blood Cell Freely Suspended in a Simple Shear Flow. - D. BARTHES-BIESEL - Université de Technologie de Compiègne BP. 23 60206 Compiègne. - B. GUERLET - Groupe de Recherche de Biophysique et d'Hémorhéologie de Nancy, CRTS 54500 Vendœuvre.

[33] SKALAK R., ZARDA P.R. et CHIEN S. - Theory of Rouleau Formation. - R. SKALAK et P.R. ZARDA, Department of Civil Engineering and Engineering Mechanics. - S. CHIEN, Department of Physiology Columbia University, New-York, N.Y. 10027. 
[34] SESHADRI V. et JAFFRIN M.Y. - Anomalous Effects in the Flow of Blood Through Narrow Tubes: A Model. - U.T.C., Dept. Génie Biologique, Compiègne 60206

[35] KENNER T., HINGHOFER-SZALKAY H, et LEOPOLD H. - Experimetal Observation and Interpretation of Capillary Fluid Shifts Using a new Method. - Physiologisches Inst. A-8010 Graz, Hozartgasse 14 and Inst. Physikalische Chemie, A-8010 Graz, Heinrichstrasse 28.

[36] SYROTA A., BUI-XUAN B., THEVEN D., GENAIN C., VALLOIS J.M. et POCIDALO J.J. - Water and Small Molecule Transport in the Microcirculation. Determination of the Thermodynamic Coefficients in Pulmonary Capillary Membrane. - Hôpital Claude Bernard 10, Av. de la Porte d'Aubervilliers 75019 Paris.

[37] PERONNEAU P. - Flow and Velocity Measurements in Blood Vessels. - Centre d'Etudes des Techniques Chirurgicales CNRS - Hôpital Broussais Paris.

[38] SIOUfFI M., PERONNEAU P. et PELISSIER R. - Modifications of Flow Patterns Induced by a Vadcular Ste. nosis. - M. SIOUFFI, Institut de Mécanique des Fluides, Université d'Aix-Marseille - P. PERONNEAU - Hôpital Broussais, Paris

[39] CLARK C. - Turbulence Measurements in a model of Aortic Stenosis. - Department of Engineering Science, University of Oxford, England

[40] TOMM D. - Model Investigation of Noise Generation in Vessel Stenosis. - Aerodynamisches Institut der RWHT Aachen, Aachen, Germany

[41] DOFFIN J., CHAGNEAU F., BORZEIX J. et RIPERT G. - Modélisation de l'Ecoulement Sanguin dans les Variations de Section Arterielle. - Faculté des Sciences de Poitiers, Laboratoire de Physique et Mécanique des Fluides, Equipe III du LA n 191 du CNRS, Av. du Recteur Pineau, 86022 Poitiers Cedex.

[42] FORESTIER B., GRASSI J.P., IMBERT P., PELISSIER R. et ROUX B. - High Order Numerical Method for the
Study of the Oscillating Flow in a Plane Constricted TwoDimensional Conduit. - Institut de Mécanique des Fluides - Université Aix-Marseille

[43] SCHWERDT H. et CONSTANTINESCO A. - The effect of Non Linear Anisotropic Elastic Properties of Arteries on Their Dynamic Radial Distensibility. - Groupe de Biomécanique - Institut de Physique Biologique,4 rue Kirschleger F. 67085 Strasbourg Cedex.

[44] SANDMANN W., PERONNEAU P., XHAARD M. et SCHWEINS G. - Mesurement of Blood Flow, Blood Velocity Profiles and Turbulence in Arterial Surgery. - Surgical clinic A (Head : Prof. Dr. med. K. Krener) of the University of Dusseldorf, Germany. - Centre d'Etudes des Techniques Chirurgicales (Head : Dr. Ing. P. Peronneau and Dr. med. J. HINGLAIS), CNRS., Paris

[45] BUTLER P.A. et SOMMERVILLE P.G. - The Use Hydraulic Imput Impedance in the evaluation of Obliterative Arterial Disease. - Sussex University, Brighton, England

[46] MASSON B., BASSE-CATHALINAT B., ARNAUD D., BAQUEY Ch., JANVIER G., TORRIELLI R. et GODARD - Etude des Modifications du Retour Veineux d'un Membre sous l'Influence d'un Shunt ArterioVeineux. - B. MASSON, Institut de Recherche en Chirurgie Expérimentale - Université de Bordeaux II, Place de la Victoire, Bordeaux. - B. BASSE-CATHALINAT, D. ARNAUD et Ch. BAQUEY, Service de Médecine Nucléaire du Pr. BLANQUET, USN Tastet Girard- Domaine de Pellegrin, Place Amélie Raba-Léon - Bordeaux. Unité de Recherches sur les Applications Médicales et Biologiques des Isotopes Radioactifs (INSERM U.53) Domaine de Carreire, rue Camille St Saêns,Bordeaux. - G. JANVIER et $R$. TORRIELLI, Service du Pr TINGAUD, Hôpital Tastet Girard, Domaine de Pellegrin. - GODARD, Département de Mesures Physiques, IUT Bordeaux Talence.

\section{Discussion}

Président : M.Y. JAFFRIN

M. le Président JAFfrin présente $M$. le Professeur COMOLET, spécialiste de la mécanique expérimentale des fluides, à l'Université Paris VI à Orsay.

"... Il va nous donner un bref compte-rendu du colloque "Euromech" sur la dynamique cardiovasculaire et respiratoire qui s'est tenu à Compiègne du 5 au 8 Septembre dernier. M. COMOLET a été l'un des organisateurs de cette réunion ; il est donc particulièrement qualifié pour en parler.

M. COMOLET présente son exposé.

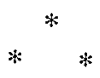

M. le Président remercie M. COMOLET de son exposé très complet et très intéressant et se félicite du succès remporté par ce colloque de Compiègne, le $92^{\mathrm{e}}$ de la série "Euromech", commencée il y a 12 ans: avec 115 participants la réunion débordait sensiblement la limite de 100 qui s'impose traditionnellement à cette manifestation. Il précise enfin que le prochain colloque "Euromech" aura lieu à Zurich en 1979, en Août sans doute, organisé par le Professeur ANLIKER: les suivants sont actuellement envisagés en 1981 et 1983 aux Pays-Bas et en Suède.

Il ouvre la discussion.

M. COMOLET précise que les colloques "Euromech" ont été fondés il y a 12 ans, celui de Compiègne portait le numéro 92 , le $100^{\mathrm{e}}$ est annoncé.
M. ODDOU remarque l'évolution des sujets traités par les colloques "Euromech": l'hydrodynamique macroscopique a progressivement céđé le pas à la microhydrodynamique et même à l'hydrodynamique moléculaire, comme à Compiègne.

M. CAZENAVE se demande si cette évolution signifie que l'hydrodynamique traditionnelle est suffisamment connue ou un peu laissée de côté.

M. ODDOU pense que, concernant la grande circulation des artères, les problèmes actuels concernent plutôt le comportement mécanique de la paroi ou les relations hémorhéologiques que l'hydrodynamique.

M. COMOLET observe cependant que si la circulation artérielle a été beaucoup étudiée, de nombreux domaines demeurent à approfondir par des équipes scientifiques: circulation veineuse, toute la mécanique collabable, la mécanique "pulmonaire"...

M. KOPP remarque d'une façon générale que les recherches tendent à s'orienter vers l'étude des systèmes intégrant l'interaction de propriétés chimiques, physiologiques etc. d'une part, et de phénomènes purement mécaniques.

Sur une question du Docteur SEBBAN, M. COMOLET puis M. KOPP précisent que la méthode d'estimation de l'élasticité artérielle mise au point́ à Strasbourg - groupe Constantinesco - a été appliquée en milieu hospitalier avec des mesures par sonde in vivo, donc traumatisantes, mais que des procédures complètement atraumatiques sont à l'essai. 